\title{
Protocolo com nove dias de progesterona para inseminação artificial em tempo fixo em vacas taurinas adaptadas ao clima tropical
}

\author{
[Protocol with nine days of progesterone for fixed-time artificial insemination in Bos taurus cows \\ adapted to the tropical weather] \\ R. Santos ${ }^{1}$, R.D. Tortorella ${ }^{1}$, E.A. Barbosa ${ }^{1}$, H.C.A. Teixeira ${ }^{1}$, \\ M.A. Paiva Neto ${ }^{2}$, A.F. Ramos ${ }^{2 *}$ \\ ${ }^{1}$ Universidade de Brasília - Brasília, DF \\ ${ }^{2}$ Embrapa Recursos Genéticos e Biotecnologia - Brasília, DF
}

\begin{abstract}
RESUMO
Três experimentos foram realizados para adaptar um protocolo de sincronização de estro e da ovulação para ser utilizado em programas de inseminação artificial em tempo fixo (IATF) em vacas taurinas tropicalmente adaptadas. No Exp. 1 (crossover), vacas pluríparas Curraleiro Pé-Duro $(n=12)$ receberam um dispositivo intravaginal contendo $1 \mathrm{~g}$ de $\mathrm{P} 4$ por oito dias e $2 \mathrm{mg}$ de BE intramuscular (IM) no momento da inserção do dispositivo (dia 0 ). No dia da remoção do dispositivo (dia 8), as fêmeas receberam $150 \mu \mathrm{g}$ de D-cloprostenol sódico e 300UI de gonadotrofina coriônica equina (eCG) IM, sendo, então, divididas aleatoriamente para receber $1 \mathrm{mg}$ de BE no dia 8 (BE8) ou $1 \mathrm{mg}$ de BE no dia 9 (BE9). A aplicação de BE no D9 atrasou a ovulação em aproximadamente 15 horas $(\mathrm{P}<0,05)$. No Experimento 2, foram avaliados protocolos com oito (P4D8) e nove dias (P4D9) de exposição à progesterona, resultando em parâmetros de desenvolvimento folicular e luteal semelhantes entre os tratamentos $(\mathrm{P}>0,05)$. No Experimento 3, os protocolos hormonais de IATF BE8 e P4D9 foram testados para a taxa de prenhez, alcançando $23 \%$ $(10 / 43)$ e $20 \%$ (9/45), respectivamente $(P>0,05)$. Embora o grupo P4D9 tenha mostrado avanço na proporção de animais que responderam ao protocolo quando comparado ao protocolo BE8, este não se refletiu em melhora na taxa de prenhez.
\end{abstract}

Palavras-chave: curraleiro pé-duro, fertilidade, sincronização, ovulação, reprodução

\begin{abstract}
Three experiments were performed to adapt a synchronization protocol of estrus synchronization and ovulation to be used in fixed time artificial insemination programs (FTAI) in tropically adapted Bos taurus cows. In Exp. 1 (crossover) multiparous Curraleiro Pé-Duro cows $(n=12)$ received an intravaginal device containing $1 \mathrm{~g}$ of $P 4$ for 8 days and $2 \mathrm{mg}$ of $E B$ at the time of device insertion (Day 0 ). On the P4 device removal (Day 8) females received $150 \mathrm{~g}$ of D-cloprostenol Sodic and $300 I U$ of equine chorionic gonadotropin (eCG). Then, they were randomly divided to receive Img of EB on Day 8 (EB8) or on Day 9 (EB9). EB9 delayed ovulation approximately 15 hours $(P<0.05)$. In Exp. 2, protocols using progesterone for eight (P4D8) or nine days (P4D9) were evaluated, resulting in similar parameters of folicular and luteal development $(P>0.05)$. In Exp. 3, EB8 and P4D9 protocols were used to evaluate the pregnancy rate, reaching 23\% (10/43) and 20\% (9/45), respectively (P>0.05). Although P4D9 protocol has shown improvement in proportion of animals that responded to the protocol when compared to EB8 protocol, it was not able to improve pregnancy rate.
\end{abstract}

Keywords: curraleiro pé-duro, fertility, ovulation, reproduction, synchronization

Recebido em 3 de janeiro de 2017

Aceito em 22 de junho de 2017

*Autor para correspondência (corresponding author)

E-mail: alexandre.floriani@embrapa.br 


\section{INTRODUÇÃO}

Os bovinos localmente adaptados, como o Curraleiro Pé-Duro, são oriundos dos primeiros animais trazidos das regiões ibéricas pelos colonizadores cinco séculos atrás. Esses animais foram selecionados naturalmente, desenvolvendo características de adaptação e resistência às diversas condições ambientais. Nesse sentido, apresentam potencial para serem criados em locais considerados desfavoráveis a outras raças, como nas regiões semiáridas (Mariante et al., 2008). Algumas características foram desenvolvidas durante esses anos de seleção natural, como a resistência a altas temperaturas, baixa umidade, doenças em geral, ectoparasitas e endoparasitas. Apesar disso, o número desses animas vem diminuindo com a maior utilização de raças comerciais que passaram por programas de melhoramento genético (Fioravanti et al., 2011).

Protocolos hormonais de inseminação artificial em tempo fixo (IATF), desenvolvidos a partir do conhecimento da dinâmica folicular, vêm sendo aplicados com resultados variáveis em raças taurinas e zebuínas (Sá Filho et al., 2013; Tortorella et al., 2013). Esses protocolos hormonais foram desenvolvidos levando em consideração diferenças fisiológicas entre essas duas sub-espécies (Castilho et al., 2007; Ginther et al., 1997), com essas diferenças também sendo observadas dentro da mesma raça, quando avaliados animais em diferentes condições fisiológicos, como constatado por Tortorella et al. (2017),m ao trabalhar com vacas e novilhas Curraleiro Pé-Duro.

Segundo Tortorella et al. (2016), o tamanho do folículo ovulatório das fêmeas Curraleiro PéDuro apresenta menor tamanho quando comparado ao de outras raças taurinas. Informações como essa, sobre o padrão de crescimento folicular, afetam a escolha do protocolo de manipulação hormonal a ser utilizado, e esse deve garantir bons índices reprodutivos.

O conhecimento e a utilização do protocolo de sincronização de estro ideal podem melhorar a fertilidade das fêmeas, auxiliando, por exemplo, $\mathrm{o}$ retorno à ciclicidade de vacas em anestro e aumentando o número de descendentes desses animais. Segundo Aerts e Bols (2010), o uso de técnicas de reprodução assistida pode auxiliar no processo de seleção artificial e na preservação de animais em risco de extinção.

Raças taurinas adaptadas ao clima tropical, como a Curraleiro Pé-Duro, apresentam características adaptativas importantes, como a baixa exigência nutricional, tornando-se opção para regiões do país onde a ausência de chuvas e a presença de pastagens de baixa qualidade são situação comum. Entretanto, pouco se sabe sobre a fisiologia reprodutiva e a eficiência de protocolos hormonais de IATF para essas fêmeas, informações necessárias para a escolha do melhor protocolo a ser utilizado. Dessa forma, o objetivo do presnete estudo foi avaliar $o$ desenvolvimento folicular e luteal de vacas taurinas tropicalmente adaptadas da raça Curraleiro Pé-Duro submetidas a protocolos hormonais de IATF com diferentes tempos de exposição à progesterona e indução da ovulação em diferentes dias do protocolo.

\section{MATERIAL E MÉTODOS}

Todos os procedimentos foram aprovados pela Comissão de Ética no Uso Animal da Embrapa Recursos Genéticos e Biotecnologia (protocolo Ceua/Cenargen 07/2013).

O trabalho foi composto por três experimentos. O Experimento 1 (Exp. 1) foi realizado no Campo Experimental Sucupira, Brasília-DF, Brasil, onde a altitude varia de 1050 a 1250 metros. O clima predominante é o Koppen Aw, indicando inverno seco (umidade relativa pode chegar a 10\%) e verão chuvoso. Os Experimentos 2 e 3 foram conduzidos em uma fazenda do município de Cocalzinho-GO, Brasil, que possui condições climáticas semelhantes às da propriedade do Exp. 1.

Previamente aos experimentos, todas as fêmeas foram submetidas a exame ginecológico e avaliação ultrassonográfica por via transretal (Mindray 2200 Vet, Shenzhen, China; 7,5MHz), para determinação de ausência de doenças ou anormalidades no trato reprodutivo.

Os animais foram mantidos em pastagem Brachiaria decumbens, com mineralização e água a vontade. No Exp. 1, foram utilizadas 12 vacas Curraleiro Pé-Duro cíclicas, pluríparas, não lactantes (último parto havia seis meses), 
entre três e oito anos e com condição corporal (CC) média 3 (1- muito magra e 5- muito gorda; Wildman et al., 1982).

As fêmeas foram divididas em dois grupos de tratamentos hormonais: BE8 e BE9, e o experimento foi delineado em modelo crossover para permitir que todos os animais participassem de todos os grupos. Todos os animais receberam por oito dias um dispositivo intravaginal contendo $1 \mathrm{~g}$ de P4 (Sincrogest $^{\circledR}$, Ouro Fino, Cravinhos, São Paulo, Brasil) e 2mg de BE IM
(Gonadiol $^{\circledR}$, Intervet Schering, Cotia, São Paulo, Brasil) no momento da inserção do dispositivo (dia 0). No momento da retirada do dispositivo (dia 8), foram aplicadas $0,150 \mathrm{mg}$ de Dcloprostenol sódico $\left(\mathrm{PGF}_{2 \alpha}\right.$; Prolise ${ }^{\circledR}$, Tecnopec LTDA, São Paulo, Brasil), 300UI de gonadotrofina coriônica equina (eCG; Novormon $^{\circledR}$ 5000UI, Schering-Plough, São Paulo, Brasil), e os animais foram divididos aleatoriamente para receber $1 \mathrm{mg}$ de BE no dia 8 $(\mathrm{BE} 8, \mathrm{n}=12)$ ou no dia 9 (BE9, $n=12$; Fig. 1$)$.

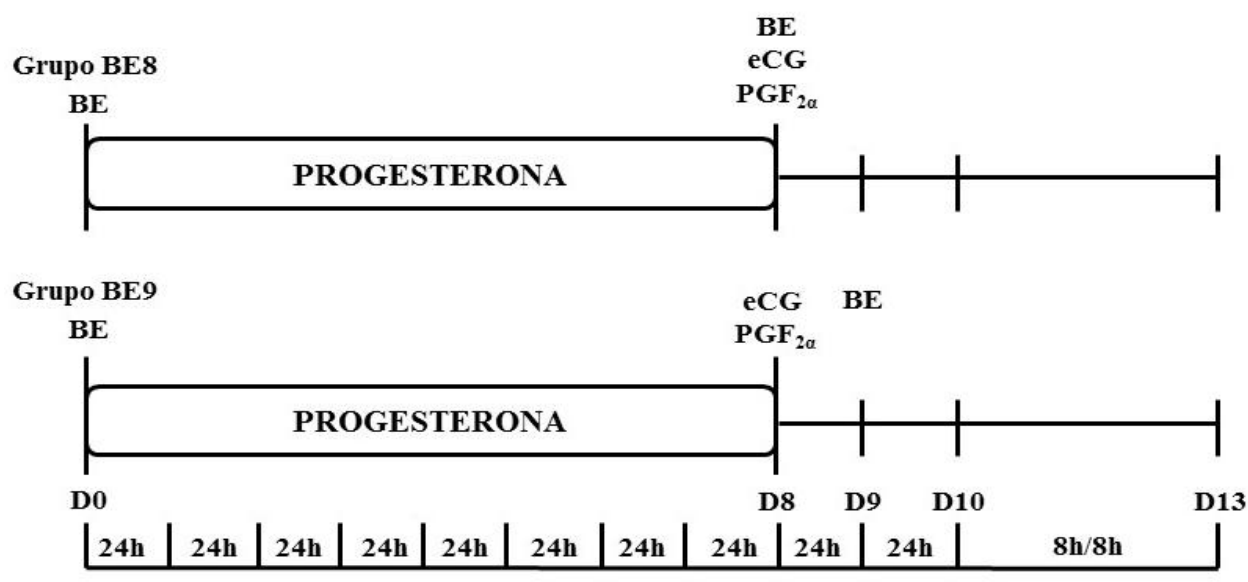

US

Figura 1. Protocolos de sincronização de estro em vacas taurinas tropicalmente adaptadas com oito dias de exposição à progesterona e indução da ovulação com benzoato de estradiol (BE) no oitavo (Grupo BE8) ou no nono dia (Grupo BE9).

O crescimento folicular foi avaliado a cada 24 horas do dia 0 (D0) até o dia 10 (D10) por ultrassonografia transretal. Todos os folículos com tamanho maior que $3 \mathrm{~mm}$ foram mensurados. O tamanho folicular foi determinado pela média dos dois maiores diâmetros de cada folículo. A partir do D10 até o D13, o folículo dominante (FD) foi avaliado a cada oito horas até a identificação da ovulação. O momento da ovulação foi determinado pelo desaparecimento do FD (>10mm) em dois exames consecutivos, seguido pela formação de um corpo lúteo (CL) em seu local. O intervalo entre a retirada da $\mathrm{P} 4$ e a ovulação foi calculado em função da média entre o momento (horas) da última visualização do FD e da avaliação seguinte, na qual não foi possível sua visualização.

O volume do CL foi mensurado 10 dias após a ovulação, utilizando-se a fórmula $\mathrm{V}=4 / 3 \times \pi \times$
$\mathrm{R}^{3}$, na qual $\mathrm{R}=(\mathrm{Da} / 2+\mathrm{Db} / 2) / 2$ e $\mathrm{Da}$ e $\mathrm{Db}$ são os diâmetros perpendiculares do CL. Na existência de uma cavidade no CL, seu volume foi calculado pela mesma fórmula e subtraído do volume total do CL.

No Experimento 2 (Exp. 2), foram utilizadas vacas Curraleiro Pé-Duro cíclicas, pluríparas, não lactantes, entre três e oito anos e com condição corporal (CC) média 3. As fêmeas foram divididas em dois grupos de tratamentos hormonais: P4D8 e P4D9, em que todos os animais participaram de todos os grupos (crossover). O grupo P4D8 $(\mathrm{n}=11)$ recebeu $\mathrm{O}$ mesmo tratamento hormonal que o grupo BE9 do Exp. 1. Já no grupo P4D9 $(\mathrm{n}=11)$, a $\mathrm{P} 4$ permaneceu por mais 24 horas (Fig. 2), sendo retirada no D9. A avaliação folicular e a luteal foram semelhantes à realizada no Exp. 1. 


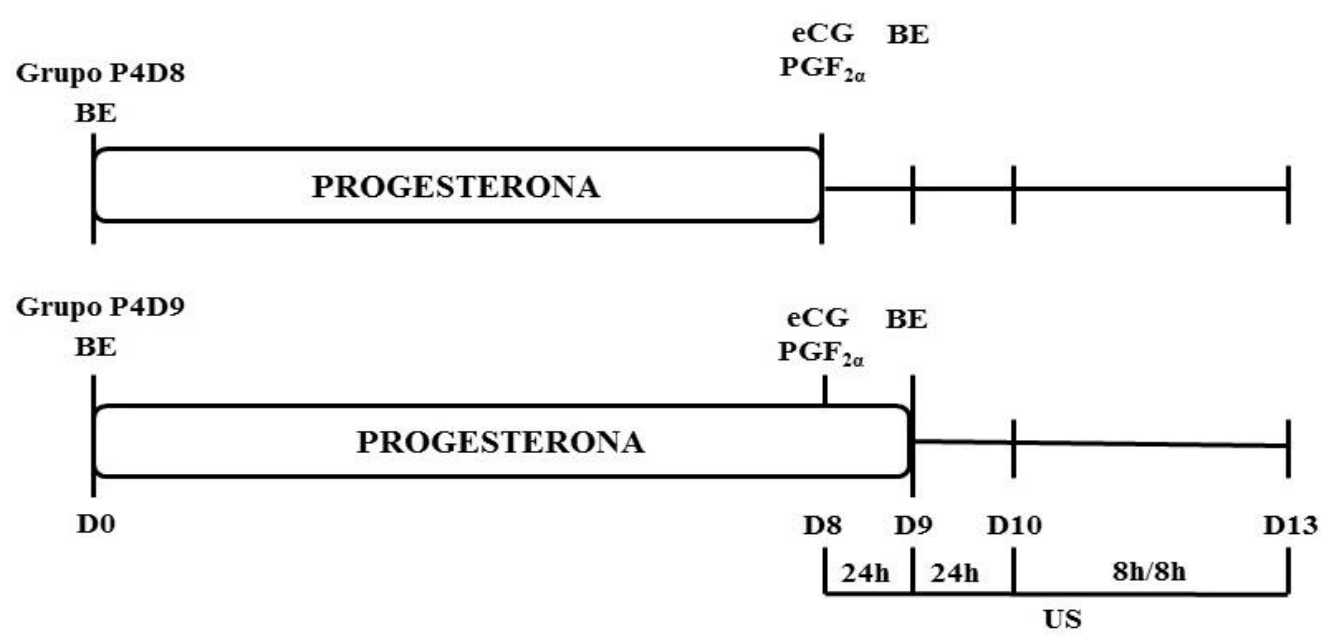

Figura 2. Protocolos de sincronização de estro em vacas taurinas tropicalmente adaptadas com oito (Grupo P4D8) ou nove dias (Grupo P4D9) de exposição à progesterona e indução da ovulação com benzoato de estradiol (BE) no nono dia.

No Experimento 3 (Exp. 3), foram utilizadas 88 vacas Curraleiro Pé-Duro cíclicas, pluríparas, lactantes (entre 60 e 90 dias pós-parto), entre três e oito anos e com condição corporal (CC) média 3. Essas fêmeas foram aleatoriamente separadas para receberem os tratamentos hormonais dos grupos BE8 e P4D9 e submetidas à IATF. No grupo BE8 $(n=43)$, as vacas foram inseminadas em tempo fixo, num intervalo de 40-42 horas após a retirada do implante de P4. Já no grupo P4D9 $(n=45)$, as inseminações ocorreram no período de 48-50 horas após a retirada do implante de P4, levando em consideração os resultados encontrados nos Experimentos 1 e 2.

Os dados foram analisados quanto à normalidade pelo teste Shapiro-Wilk. O tamanho folicular foi analisado por meio do modelo linear generalizado (GLM), utilizando-se ANOVA e contraste para comparação entre os grupos. O tempo entre a retirada da $\mathrm{P} 4$ e a ovulação e o volume do CL foi analisado também mediante a ANOVA, e as médias comparadas pelo teste de Tukey. Dados demonstrados em porcentagem foram submetidos ao teste qui-quadrado $\left(\chi^{2}\right)$ com correção de Yates. Os resultados foram apresentados como média \pm desvio-padrão (DPM) e porcentagem (taxa de ovulação e gestação). O ajuste das curvas de desenvolvimento folicular foi realizado pelo modelo de regressão linear, sendo a variável resposta o tamanho do FD, e a covariável os dias de avaliação. Para avaliar a qualidade do ajuste da curva, utilizou-se como critério o coeficiente de determinação $\left(\mathrm{R}^{2}\right)$.

\section{RESULTADOS}

No Exp. 1, não houve diferença entre os tratamentos. Consequentemente, optou-se por unir os dados para avaliar o comportamento do FD desde o dia da colocação da P4 até a ovulação (Fig. 3). A emergência da onda folicular ocorreu aos 3,3 dias após o início do protocolo, enquanto a divergência folicular ocorreu após 8,3 dias.

A presença de FD $>8 \mathrm{~mm}$ foi observada no momento da retirada da P4 em 92\% (11/12) e $83 \%$ (10/12) das fêmeas nos grupos BE8 e BE9, respectivamente $(\mathrm{P}>0,05)$. As vacas sem $\mathrm{FD}$ após a retirada da $\mathrm{P} 4$ foram removidas da análise estatística. Houve efeito do dia no crescimento folicular ao longo das avaliações $(\mathrm{P} \leq 0,001)$, entretanto os grupos não diferiram quanto a tamanho do FD, folículo ovulatório (FO), taxa de ovulação (TO) e volume luteal (Tab. 1). O intervalo entre a retirada da $\mathrm{P} 4$ e a ovulação (IO) foi menor no grupo BE8 $(58 \pm 2 \mathrm{~h} v s .73,3 \pm 3 \mathrm{~h}$; $\mathrm{P}<0,05)$. 


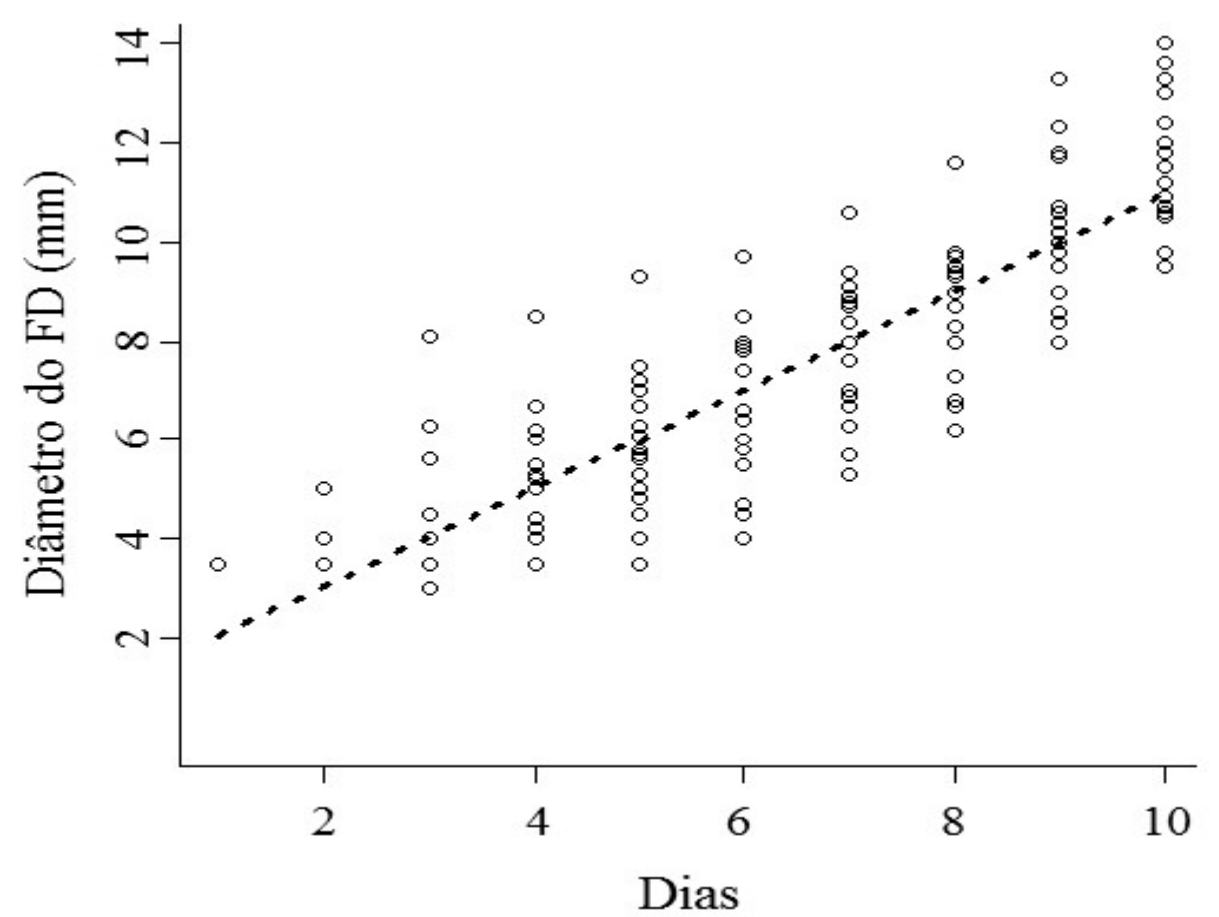

Figura 3. Análise de regressão do padrão de comportamento do desenvolvimento do folículo dominante (FD) em vacas taurinas tropicalmente adaptadas submetidas à sincronização da emergência da onda folicular com progesterona e benzoato de estradiol. $\mathrm{Y}=0,9 \mathrm{x}+1,05, \mathrm{R}^{2}=0,72$, em que $\mathrm{Y}=$ diâmetro do FD e $\mathrm{x}=$ dia da mensuração.

Tabela 1. Efeito da aplicação do BE no dia da retirada da progesterona (BE8) ou 24 horas após (BE9), em um protocolo de IATF no diâmetro do folículo dominante (D8 e D9), folículo ovulatório (FO), intervalo para ovulação (IO), taxa de ovulação (TO) e volume do corpo lúteo (CL) em vacas taurinas tropicalmente adaptadas

\begin{tabular}{ccccccc}
\hline \multicolumn{7}{c}{ Diâmetro do folículo dominante $(\mathrm{mm})$} \\
\hline Grupos & D8 & D9 & FO & IO $^{1}(\mathrm{~h})$ & TO (\%) & Volume do \\
BE8 & $8,4 \pm 0,4$ & $10 \pm 0,5$ & $11,5 \pm 0,5$ & $58 \pm 2^{\mathrm{a}}$ & $8 / 11(73)$ & $4.239 \pm 521$ \\
BE9 & $8,6 \pm 0,4$ & $10,6 \pm 0,5$ & $12,2 \pm 0,5$ & $73 \pm 3^{\mathrm{b}}$ & $10 / 10(100)$ & $3.505 \pm 487$ \\
\hline
\end{tabular}

Os dados foram apresentados como média \pm (DPM). Houve efeito do dia $(\mathrm{P}<0,001)$ no crescimento do folículo dominante, mas não um efeito de grupo e interação grupo por dia nos outros parâmetros. ${ }^{\mathrm{a}, \mathrm{b}} \mathrm{Na}$ mesma coluna, médias com letras diferentes diferem $(\mathrm{P} \leq 0,05) .{ }^{1} \mathrm{O}$ intervalo entre a retirada da $\mathrm{P} 4$ e a ovulação foi calculado em função da média entre o momento (horas) da última visualização do FO e da avaliação seguinte, na qual não foi possível sua visualização.

No Exp. 2, a presença de FD> $>\mathrm{mm}$ foi observada no momento da retirada da P4 em $72 \%(8 / 11)$ e $100 \%(11 / 11)$ das fêmeas nos grupos $\mathrm{P} 4 \mathrm{D} 8$ e $\mathrm{P} 4 \mathrm{D} 9$, respectivamente $(\mathrm{P}>0,05)$. As vacas sem FD após a retirada da $\mathrm{P} 4$ foram removidas da análise estatística. Houve efeito do dia no crescimento folicular ao longo das avaliações $(\mathrm{P} \leq 0,001)$, entretanto os grupos não diferiram quanto a tamanho do FD, folículo ovulatório (FO), intervalo para ovulação (IO), taxa de ovulação (TO) e volume luteal $(\mathrm{P}>0,05$; Tab. 2). 
Tabela 2. Efeito da permanência da progesterona por oito (P4D8) ou nove (P4D9) dias, em um protocolo de IATF no diâmetro do folículo dominante (D8 e D9), folículo ovulatório (FO), intervalo para ovulação (IO), taxa de ovulação (TO) e volume do corpo lúteo (CL) em vacas taurinas tropicalmente adaptadas Diâmetro do folículo dominante $(\mathrm{mm})$

\begin{tabular}{ccccccc}
\multicolumn{7}{c}{ Diâmetro do folículo dominante $(\mathrm{mm})$} \\
\hline Grupos & D8 & D9 & FO & $\mathrm{IO}^{1}(\mathrm{~h})$ & TO $(\%)$ & Volume do \\
P4D8 & $8,6 \pm 0,4$ & $10 \pm 0,4$ & $11,7 \pm 0,4$ & $60 \pm 4$ & $7 / 8(87,5)$ & $3.560 \pm 566$ \\
P4D9 & $8,6 \pm 0,4$ & $9,9 \pm 0,3$ & $11,3 \pm 0,3$ & $70 \pm 4$ & $10 / 11(91)$ & $4.803 \pm 524$ \\
\hline
\end{tabular}

Os dados foram apresentados como média $\pm(\mathrm{DPM})$. Houve efeito do dia $(\mathrm{P}<0,001)$ no crescimento do folículo dominante, mas não um efeito de grupo e interação grupo por dia nos outros parâmetros. ${ }^{1} \mathrm{O}$ intervalo entre a retirada da P4 e a ovulação foi calculado em função da média entre o momento (horas) da última visualização do FD e da avaliação seguinte, na qual não foi possível sua visualização.

Não houve diferença na taxa de prenhez (P>0,05; Exp. 3) entre os grupos BE8 (10/43=23\%) e P4D9 (9/45=20\%).

\section{DISCUSSÃO}

No Exp. 1, após análise de regressão foi possível observar que o início da nova onda folicular ocorre aproximadamente 3,3 dias após a colocação da $\mathrm{P} 4$, confirmando o relatado por Mapletoft et al. (2003). A associação de um dispositivo vaginal com $\mathrm{P} 4$ e a aplicação do BE IM levam à diminuição na pulsatilidade de LH e à concentração circulante de FSH, o que acaba por regredir tanto folículos pequenos quanto grandes (Siqueira et al., 2009).

As fêmeas que possuíam FD > 8mm (divergência folicular ocorre em torno de $8,5 \mathrm{~mm}$ ) no momento da retirada da $\mathrm{P} 4$ foram consideradas sincronizadas, e o protocolo padrão eficaz em iniciar uma nova onda. As vacas em que o protocolo não foi eficaz foram excluídas da análise para evitar subestimação dos resultados. $\mathrm{O}$ protocolo base contendo $\mathrm{P} 4, \mathrm{BE}$ e $\mathrm{PGF}_{2 \alpha}$ foi capaz de sincronizar em torno de $85 \%$ das fêmeas (Exp. 1 e 2), resultado semelhante aos obtidos por Tortorella et al. (2016) em animais da mesma raça, entretanto Sales et al. (2012) relataram que apenas $6,7 \%$ das fêmeas não possuíam FD > 8,5mm após a retirada da $\mathrm{P} 4$.

No momento da retirada do implante de progesterona, que ocorreu oito dias após o início dos protocolos BE8 e BE9 (Exp. 1), pôde-se verificar que parte dos animais não apresentavam folículo dominante maior que $8 \mathrm{~mm}$ de diâmetro, o que poderia reduzir a eficiência dos protocolos utilizados. Colazo et al. (2003) sugerem que a exposição à progesterona por um dia a mais no protocolo, ou seja, nove dias, levaria ao aumento do folículo dominante; isso, segundo Perry et al.
(2007), acarretaria em maiores taxas de ovulação e gestação.

O BE administrado na retirada da $\mathrm{P} 4$ (BE8) antecipou a ovulação em relação à aplicação 24 horas após a retirada da P4 (BE9). Mapletof et al. (2003) relataram que o BE leva a um pico de LH entre 16 e 20 horas após sua aplicação e que a ovulação ocorre entre 70 e 96 horas após a retirada da $\mathrm{P} 4$, semelhante ao que foi encontrado no grupo BE9.

Não houve diferença no volume do CL, o que era esperado, visto que o tamanho do FD também não diferiu. Vasconcelos et al. (2001) relataram que, quanto maior o diâmetro do FD, maior o volume do CL. Ainda, o volume do CL tem sido correlacionado positivamente com a secreção de P4 (Sartori et al., 2002).

No Exp. 2, com a manutenção do dispositivo de progesterona por nove dias (grupo P4D9), era esperado que a permanência da $\mathrm{P} 4$ por mais 24 horas e a aplicação do eCG e da $\mathrm{PGF}_{2 \alpha}$ um dia antes da retirada da $\mathrm{P} 4$ pudessem estimular o crescimento do FD e, consequentemente, a taxa de ovulação e o volume do CL. Contudo, no presente estudo, a aplicação desses hormônios apenas um dia antes da retirada da P4 (eCG e $\mathrm{PGF}_{2 \alpha}$ ) não foi suficiente para aumentar $\mathrm{o}$ estímulo ao FD.

$\mathrm{O}$ eCG tem maior eficiência em fêmeas com baixa pulsatilidade de LH, situação encontrada em animais com baixa CC (Núñez-Olivera et al., 2014). Bilbao et al. (2016) demonstraram que fêmeas com baixo CC obtiveram maior taxa de gestação quando utilizado o eCG no protocolo de IATF. Entretanto, Pegorer et al. (2011), ao 
trabalharem com novilhas Bos indicus, não observaram efeito do eCG na taxa de gestação e no tamanho do folículo dominante. $\mathrm{O}$ fato de as fêmeas desse experimento se encontrarem em boa $\mathrm{CC}$ pode ter sido uma das razões para o eCG não ter afetado o crescimento do FD. A manutenção da $\mathrm{CC}$ ao longo das estações, mesmo em condições ambientais de deficiência hídrica e alimentar, é uma das características adaptativas das raças localmente adaptadas. Bianchini et al. (2006) sugerem que o tamanho pequeno da raça seria justamente uma adaptação a condições ambientais adversas.

No Exp. 3, a permanência da $\mathrm{P} 4$ por mais 24 horas e a aplicação do eCG e da $\mathrm{PGF}_{2 \alpha}$ antes da retirada da $\mathrm{P} 4$ não aumentaram a taxa de prenhez, provavelmente porque os animais apresentavam boa condição corporal. Bilbao et al. (2016) demonstraram que o aumento no tempo de exposição à progesterona durante o protocolo de IATF aumentou o tamanho do folículo dominante em vacas com bom $\mathrm{CC}$, porém não teve efeito sobre a fertilidade, já quando utilizado em vacas de baixo escore corporal, notou-se um aumento na taxa de gestação.

Embora as taxas de ovulações encontradas nos Experimentos 1 e 2 tenham sido satisfatórios (80 a $100 \%$ ), as taxas de prenhez foram baixas em ambos os grupos quando comparadas ao encontrado na literatura, em torno de $50 \%$ (Sales et al., 2012; Sá Filho et al., 2013). A utilização de vacas em lactação no Experimento 3 pode ter contribuído para a baixa taxa de prenhez alcançada.

\section{CONCLUSÕES}

Os protocolos de sincronização de estro utilizados no trabalho demonstraram boa taxa de sincronia e ovulação. Embora observada baixa taxa de gestação das fêmeas quando inseminadas utilizando esses protocolos, os resultados quanto ao desenvolvimento folicular e à ovulação sugerem a utilização desses protocolos em programas de IATF em vacas taurinas tropicalmente adaptadas.

\section{AGRADECIMENTOS}

Os autores agradecem ao CNPq e à FAP-DF, pelo suporte financeiro, e à Rede Centro-Oeste de Pós-Graduação, Pesquisa e Inovação.

\section{REFERÊNCIAS}

AERTS, J.M.J.; BOLS, P.E.J. Ovarian follicular dynamics. A review with emphasis on the bovine species. Part II: Antral development, exogenous influence and future prospects. Reprod. Dom. Anim., v.45, p.180-187, 2010.

BIANCHINI, E.; CONCEPTA, M.; LUCCI, C.M. et al. Características corporais associadas com a adaptação ao calor em bovinos naturalizados brasileiros. Pesqui. Agropecu. Bras., v.41, p.1443-1448, 2006.

BILBAO, M.G.; MASSARA, N.; RAMOS, S. et al. Extending the duration of treatments with progesterone and equine chorionic gonadotropin improves fertility in suckled beef cows whith low body condition score subjected to timed artificial insemination. Theriogenology, v.86, p.536-544, 2016.

CASTILHO, C.; GARCIA, J.M.; RENESTO. A. et al. Follicular dynamics and plasma FSH and progesterone concentrations during follicular deviation in the first post-ovulatory wave in Nelore (Bos indicus) heifers. Anim. Reprod. Sci., v.98, p.189-96, 2007.

COLAZO, M.G.; KASTELIC, J.P.; MAPLETOF, R.J. Effects of estradiol cypionate (EC) on ovarian follicular dynamics, synchrony of ovulation, and fertility in CIDR-based, fixedtime AI programs in beef heifers. Theriogenology, v.60, p.855-865, 2003.

FIORAVANTI, M.C.S.; JULIANO, R.S.; COSTA, G.L. et al. Conservación del bovino curraleiro: cuantificación del censo y caracterización de los criadores. Anim. Genet. Resour., v.48, p.109-116, 2011.

GINTHER, O.J.; KOT, K.; KULLICK, L.J.; WILTBANK, M.C. Emergence and deviation of follicles during the development of follicular waves in cattle. Theriogenology, v.48, p.75-87, 1997. 
MAPLETOFT, R.J.; MARTÍNEZ, M.F.; COLAZO, M.G.; KASTELIC, J.P. The use for controlled internal drug release devices for the regulation of bovine reproduction. J. Anim. Sci., v.81, 28-36, 2003.

MARIANTE, A.S.; EGITO, A.A.; ALBUQUERQUE, M.S.M. et al. Managing genetic diversity and society needs. Rev. Bras. Zootec., v.37, Supl., p.127-136, 2008.

NÚÑEZ-OLIVERA, R.; CASTRO, T.; GARCÍA-PINTOS, C. et al. Ovulatory response and luteal function after eCG administration at the end of a progesterone and estradiol based treatment in postpartum anestrous beef cattle. Anim. Reprod. Sci., v.146, p.111-116, 2014.

PEGORER, M.F.; ERENO, R.L.; SATRAPA, R.A. et al. Neither plasma progesterone nor exogenous eCG affects rates of ovulation or pregnancy in fixed-time artificial insemination (FTAI) protocol for puberal Nellore heifers. Theriogenology, v.75, p.17-23, 2011.

PERRY, G.A.; SMITH, M.F.; ROBERTS, A.J. et al. Relationship between size of the ovulatory follicle and pregnancy success in beef heifers. $J$. Anim. Sci., v.85, p.684-689, 2007.

SÁ FILHO, M.F.; PENTEADO, L.; REIS, E.L. et al. Timed artificial insemination early in the breeding season improves the reproductive performance of suckled beef cows. Theriogenology, v.79, p.625-632, 2013.

SALES, J.N.S.; CARVALHO, J.B.P.; CREPALDI, G.A. et al. Effects of two estradiol esters (benzoate and cypionate) on the induction of synchronized ovulations in Bos indicus cows submitted to a timed artificial insemination protocol. Theriogenology, v.78, p.510-516, 2012.
SARTORI, R.; ROSA, G.J.M.; WILTBANK, M.C. Ovarian structures and circulating steroids in heifers and lactating cows in summer and lactating and dry cows in winter. J. Dairy Sci., v.85, p.2813-2822, 2002.

SIQUEIRA, L.C.; OLIVEIRA, J.F.; ROVANI, M.T. et al. Effects of estradiol and progestins on follicular regression before, during, and after follicular deviation in postpartum beef cows. Theriogenology, v.71, p.614-619, 2009.

TORTORELLA, R.D.; FERREIRA, R.; DOS SANTOS, J.T. et al. The effect of equine chorionic gonadotropin on follicular size, luteal volume, circulating progesterone concentrations, and pregnancy rates in anestrous beef cows treated with a novel fixed-time artificial insemination protocol. Theriogenology, v.79, p.1204-1209, 2013.

TORTORELLA, R.D.; MODESTO, M.R.; NEVES, J.P. et al. Development of fixed-time artificial insemination protocols for locally adapted Curraleiro Pé-Duro cows. Arq. Bras. Med. Vet. Zootec., v.68, p.1159-1167, 2016.

TORTORELLA, R.D.; NOGUEIRA, G.P.; MODESTO, M.R. et al. Characterizing emergence and divergence in the first follicular wave in a tropically adapted Bos taurus breed. Theriogenology, v.88, p.9-17, 2017.

VASCONCELOS, J.L.; SARTORI, R.; OLIVEIRA, H.N. et al. Reduction in size of the ovulatory follicle reduces subsequent luteal size and pregnancy rate. Theriogenology, v.56, p.307314,2001

WILDMAN, E.E.; JONES, G.M.; WAGNER, P.E. et al. A dairy body condition scoring system and its relationship to selected production characteristics. J. Dairy Sci., v.65, p.495-501, 1982. 